\title{
Fotoluminescência em materiais com desordem estrutural
}

\section{(Photoluminescence in disordered materials)}

\author{
P. R. de Lucena', F. M. Pontes' ${ }^{2}$ C. D. Pinheiro ${ }^{3}$, E. Longo ${ }^{2}$, P. S. Pizani ${ }^{4}$, S. L.ázarón , \\ A. G. Souza ${ }^{1}$, I. M. G. dos Santos ${ }^{1}$ \\ ${ }^{1}$ LTM, Departamento de Química, CCEN, UFPB, Campus I, Cidade Universitária \\ J. Pessoa, PB, 58059-900. \\ ${ }^{2}$ LIEC,CMDMC, Departamento de Química, UFSCar \\ Rod. Washington Luiz, km 235, C.P. 676, \\ S. Carlos, SP, 13565-905. \\ ${ }^{3}$ DCEN, CFP, UFCG, Campus de Cajazeiras \\ Cajazeiras, PB, 58.900-000. \\ potylucena@hotmail.com
}

\begin{abstract}
Resumo
A grande maioria dos estudos fotoluminescentes foram realizados com materiais cristalinos, cuja emissão ocorre em temperaturas criogênicas, limitando a aplicação destes materiais. Por sua vez, determinados sólidos com alta densidade de defeitos (amorfos), como titanatos, tungstatos, zirconatos, entre outros, apresentam fotoluminescência à temperatura ambiente, semelhante à do silício amorfo. Essa descoberta levou à necessidade de se desenvolver novos modelos para explicar o comportamento desses materiais, mais adequados ao estudo desses materiais. Esta revisão tem como objetivo explicar os resultados de fotoluminescência, utilizando uma abordagem teórico-experimental, com base nas recentes pesquisas desta propriedade em óxidos amorfos.
\end{abstract}

Palavras-chave: fotoluminescência, titanato, óxidos amorfos, gap de energia.

\begin{abstract}
The majority of the studies about photoluminescence had been carried out with crystalline materials, whose emission occurs at cryogenic temperatures, limiting the application of these materials. In turn, some amorphous solids, as titanates, tungstates, zirconates, among others, present photoluminescence at room temperature, seemed with silicon. This discovery led to the necessity of developing new models to explain the behavior of these materials. This revision intends to elucidate the results on photoluminescence, using a theoretical-experimental treatment, on the basis of the recent researches of this property in amorphous oxides.
\end{abstract}

Keywords: photoluminescence, titanate, amorphous oxides, energy gap.

\section{INTRODUÇÃO}

Da luz verde dos vaga-lumes aos tubos catódicos da televisão, passando pelas placas de sinalização e pela identificação de manchas de sangue, a luminescência é amplamente utilizada em diversas áreas do nosso cotidiano.

O termo luminescência é utilizado como uma generalização do fenômeno. Há vários tipos de luminescência, que diferem entre si pela energia utilizada para a excitação [1], como, por exemplo, a eletroluminescência, a catodoluminescência, a bioluminescência, a luminescência química, e a fotoluminescência.

As emissões luminescentes envolvem transições eletrônicas entre os estados eletrônicos característicos da substância emissora. O espectro de emissão, para muitas substâncias, independe da natureza da fonte de excitação. O fenômeno luminescente é essencialmente uma emissão espectroscópica [1].

De um modo geral, a fotoluminescência é um fenômeno ótico produzido quando um material é excitado e exibe uma emissão de onda eletromagnética na forma de fótons. A onda eletromagnética irradiada por fotoluminescência tem, em regra, um comprimento de onda maior do que a onda eletromagnética que causou esta luminescência.

Os primeiros estudos fotoluminescentes foram realizados com materiais cristalinos, cuja emissão ocorre em temperaturas criogênicas, limitando a aplicação destes materiais [2]. O interesse em propriedades fotoluminescentes 
em materiais amorfos se deu a partir dos anos 90, devido à descoberta de luz sintonizável em silício poroso [3]. No entanto, a origem do estudo sistemático de fotoluminescência tornou-se um tópico polêmico, pois surgiram muitos modelos baseados nas observações específicas da microestrutura do material e da natureza espectroscópica do processo de emissão de luz [4].

Recentemente, óxidos amorfos, com propriedades fotoluminescentes à temperatura ambiente, foram obtidos utilizando-se o método Pechini $[5,6]$. Este método proporciona a obtenção de materiais nanométricos, com elevada homogeneidade química. Nestes óxidos, a fotoluminescência é um fenômeno intimamente associado às transições eletrônicas entre as bandas de valência e de condução. A estrutura de bandas é definida pelo acoplamento entre as funções de onda que definem as energias dos estados eletrônicos dos átomos de uma rede. Estes estados possuem energias muito próximas e são limitados pela banda de valência e pela banda de condução, que na teoria dos orbitais moleculares, correspondem ao estado fundamental e excitado, respectivamente [7].

Embora o potencial de redes cristalinas ocupe um lugar dominante no desvio da estrutura de bandas, existem evidências experimentais e bases teóricas para estruturas eletrônicas em semicondutores amorfos [6] e vidros [8]. O transporte de elétrons não muda drasticamente quando a ordem a longo alcance é destruída [9].

\section{Papel dos dopantes nos estados de energia.}

\section{Mecanismos de excitação e emissão}

Na Fig. 1, estão ilustrados os processos de excitação e de emissão para um material hipotético, com a representação esquemática dos níveis de energia. $\mathrm{E}_{0}$ é o estado fundamental e de $E_{1}$ a $E_{5}$, estão representados os estados excitados. À baixas temperaturas, na ausência de uma energia de excitação,

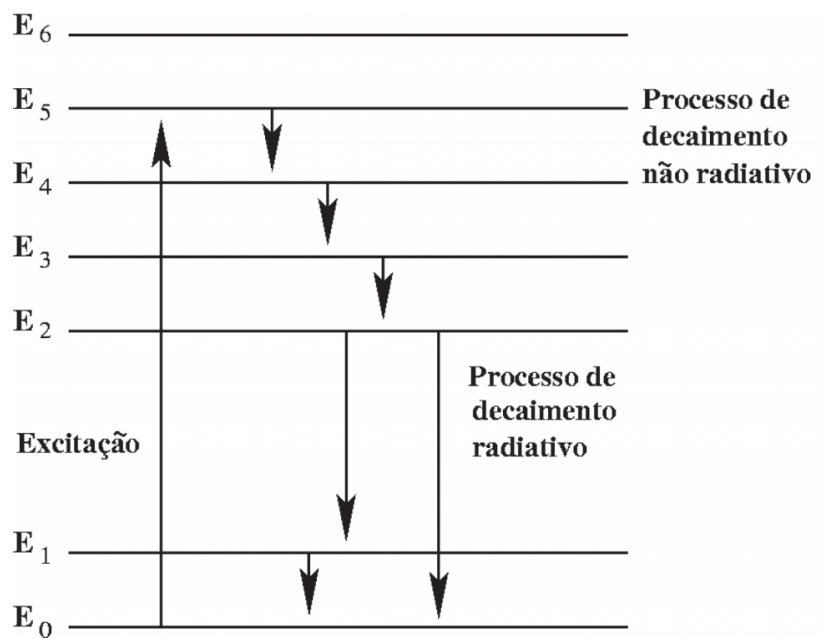

Figura 1: Processo de excitação e emissão para um material hipotético [10]. [Figure 1: Process of excitation and emission for a hypothetical material [10].] só o nível $\mathrm{E}_{0}$ é ocupado. Após a excitação, elétrons são ativados para o nível $\mathrm{E}_{5}$. Os intervalos de energia entre os níveis adjacentes de $E_{2}$ ao $E_{5}$ são pequenos, enquanto que o intervalo entre $\mathrm{E}_{2}$ e $\mathrm{E}_{1}$ é grande. Se o intervalo entre um nível excitado e o mais próximo adjacente é pequeno, o material excitado tende a apresentar um decaimento não radiativo pela emissão de fônon, liberando energia na forma de calor. A radiação eletromagnética, que é resultante de um decaimento radiativo de um nível eletrônico superior para o estado fundamental, pela emissão de um fóton, só ocorre quando o intervalo para o nível adjacente mais baixo está acima de um valor crítico. Quando o elétron é excitado para o nível $\mathrm{E}_{5}$, este perde energia até atingir o nível 2. Como o intervalo dos níveis 2 e 1 está acima do valor crítico, então o material decai radiativamente do nível 2, emitindo um fóton, alcançando o nível 1 ou 0 . Se o material decai radiativamente para o nível 1 , este então, decai não radiativamente por intermédio do pequeno intervalo para o estado fundamental [10].

\section{Efeito das impurezas}

A fotoluminescência, na maioria dos sólidos inorgânicos, envolve impurezas (denominadas dopantes) ou defeitos estruturais (vacâncias). Estas imperfeições são do tipo atômica e/ou molecular, sendo que suas características dependem da natureza e estrutura da imperfeição e dos estados eletrônicos do sólido [2].

O fenômeno fotoluminescente pode ser potencializado pelo efeito de dopantes [6], que podem criar, dependendo da energia do seu campo ligante, novos estados eletrônicos, que podem facilitar a transição de elétrons de um nível mais energético para um menos energético, emitindo energia na forma de fótons, ou seja, um decaimento radioativo. Por outro lado, os dopantes podem diminuir a emissão de fótons, criando estados eletrônicos responsáveis pelo decaimento não-radioativo, provocando o que é conhecido como envenenamento [7]. Um outro importante fator que influencia as propriedades óticas de muitos sólidos inorgânicos é o tamanho dos raios dos cátions da rede.

Os estados eletrônicos, responsáveis pelas transições eletrônicas, são criados entre a banda de valência e a de condução, a partir do desdobramento de orbitais do átomo dopante mantido em um campo ligante. Com base nesta teoria, foram desenvolvidos os diagramas de energias dos desdobramentos do estado fundamental de metais de transição, considerando as interações dos seus orbitais e sua relação com o campo ligante octaédrico e tetraédico a que estão submetidos [11]. Estes diagramas são conhecidos como diagramas configuracionais de Tanabe e Sugano.

As impurezas que ocasionam defeitos na estrutura cristalina de semicondutores podem ser classificadas em três categorias: doadoras, aceptoras e isoeletrônicas. Doadoras são substâncias que podem doar um de seus elétrons para a banda de condução de um semicondutor. A energia necessária para esta transição do nível doador para a banda de condução $\left(E_{D}\right)$ está representada esquematicamente na Fig. 2. Se as impurezas presentes são capazes de retirar um elétron da 


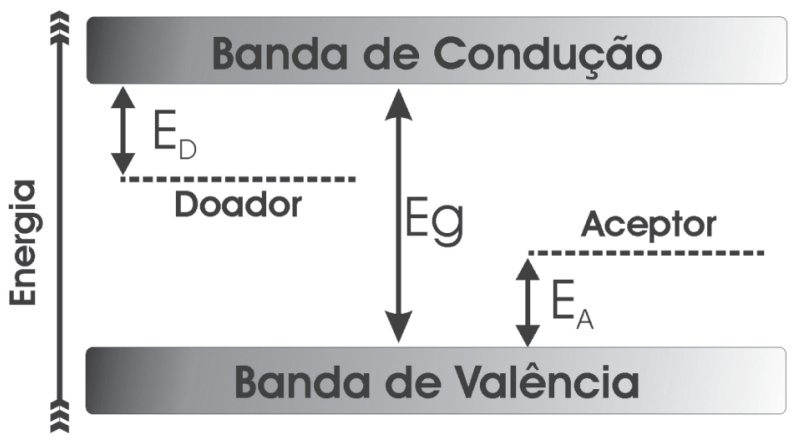

Figura 2: Representação dos níveis de energia gerados por impurezas doadoras e receptoras em semicondutor. Eg é a energia do "gap" de banda [10].

[Figure 2: Representation of the energy levels generated by donor and acceptor impurities in semiconductor. Eg is the energy of band gap [10].]

banda de valência de um semicondutor e deixar um buraco na mesma, são denominadas aceptoras. A energia necessária para a remoção $\left(\mathrm{E}_{\mathrm{A}}\right)$ está ilustrada na Fig. 2. Impurezas isoeletrônicas são substâncias que não contribuem para a condutividade elétrica de um semicondutor, mas podem influenciar nas propriedades luminescentes do material, atuando como centros de recombinação de elétrons excitados e buracos [10].

De um modo geral, as transições eletrônicas são regidas por regras de seleção de Laporte e de Spins que, por sua vez, são regidas pelas regras de Hund e são classificadas como transições permitidas ou proibidas, sendo que esta última ocorre muito raramente e possui uma intensidade muito menor que as transições permitidas [12].

As reações de substituição de cátions muitas vezes não consideram a polarização de cargas ao longo da rede. Esta polarização forma defeitos eletrônicos na estrutura. A presença desses defeitos é indesejável, mesmo em baixas concentrações, pois pode diminuir a emissão fotoluminescente de muitos materiais. Este problema pode ser contornado pela compensação de cargas. Em um estudo comparativo [13], foi concluido que os óxidos preparados pelo método sol-gel com a compensação de cargas apresentaram um ganho de aproximadamente $250 \%$ na intensidade da emissão fotoluminescente. Foi mostrado que o desempenho óptico de titanatos de cálcio dopados com praseodímio depende dessa compensação de cargas.

Cabe ressaltar que as emissões fotoluminescentes produzem informações estruturais de características completamente diferentes das obtidas por difração de raios $\mathrm{X}$ ou de nêutrons. Esta última detecta ordem a longo alcance, isto é, a estrutura cristalina total. A fotoluminescência produz somente informações do íon em relação a sua vizinhança, e pode, dessa forma investigar a ordem a curto alcance [7].

\section{Fotoluminescência em óxidos cristalinos}

Os materiais cristalinos possuem uma estrutura organizada à longo alcance com uma repetição periódica. No princípio todo estudo fotoluminescente era baseado em sólidos cristalinos, como a sheelita $\left(\mathrm{CaWO}_{4}\right)$, que foi largamente utilizada em lâmpadas perdendo a sua posição de líder no mercado para os materiais dopados com terras raras, mais atrativos às indústrias da época. A exploração de novas propriedades em materiais cristalinos, sobretudo as óticas, ficou limitada pelas baixas temperaturas requeridas $\left(-5\right.$ a $\left.-70{ }^{\circ} \mathrm{C}\right)$, o que de certa forma inviabiliza a utilização destes materiais em muitos dispositivos que atuam em temperatura ambiente.

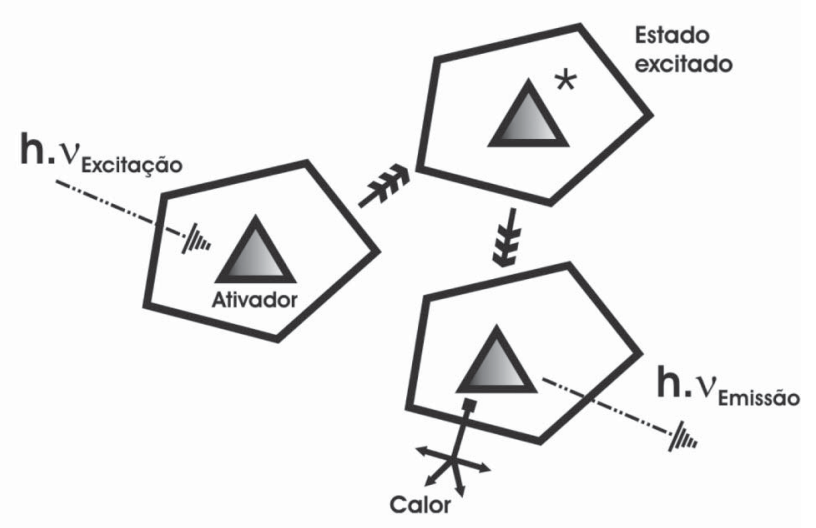

Figura 3: Processo de emissão de um material cristalino. [Figure 3: Process of emission of a crystalline material.]

Analisando a Fig. 3 que ilustra a estrutura de um sólido cristalino. Quando se incide um certo tipo de radiação eletromagnética (radiação de excitação) no material cristalino, a radiação é absorvida pelo centro luminescente ou ativador produzindo o estado excitado. $\mathrm{O}$ estado excitado retorna ao estado fundamental perdendo energia. Em que parte desta energia é dissipada e se converte em calor (processo não radioativo) e, porém a outra parte é aproveitada no processo de emissão de luz. Em alguns sólidos (Fig. 4) a energia incidente é absorvida por um sensitizador e transferida para o ativador que então atingirá o estado excitado produzindo, no retorno ao estado fundamental, radiação eletromagnética. É o que acontece quando se incide radiação ultravioleta no $\mathrm{Ca}_{5}\left(\mathrm{PO}_{4}\right)_{3} \mathrm{~F}: \mathrm{Sb}^{3+}, \mathrm{Mn}^{2+}$, um material utilizado em lâmpadas. O íon $\mathrm{Mn}^{2+}$ não é excitado diretamente, a energia de excitação é transferida do $\mathrm{Sb}^{3+}$ (sensitizador) para o $\mathrm{Mn}^{2+}$ (ativador).

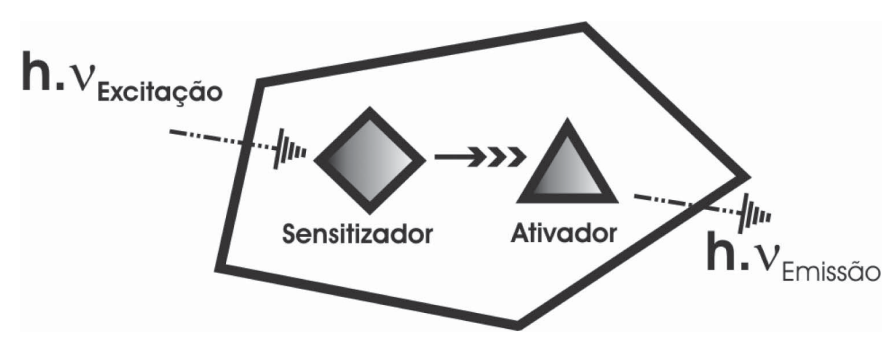

Figura 4: Processo de emissão de um material utilizando um sensitizador.

[Figure 4: Process of emission of a material using a sensitizer.] 


\section{Fotoluminescência em óxidos amorfos}

Nos anos 90, observou-se luminescência em titanatos semicondutores [14]. Nestes materiais, a emissão fotoluminescente, à baixa temperatura, está associada à presença de excitons no poliedro de coordenação octaédrica $\mathrm{TiO}_{6}$. Por sua vez, o fenômeno da fotoluminescência em titanatos amorfos, à temperatura ambiente, ainda não foi completamente modelado. Várias propriedades destes titanatos amorfos têm sido descritas, inclusive o fato que as emissões de ondas fotoluminescentes estão relacionados ao comprimento de onda de excitação e que os fenômenos fotoluminescentes estão relacionados ao estado amorfo $[15,16]$.

As propriedades óticas de semicondutores amorfos são caracterizadas pela presença de uma cauda de absorção da energia do fóton versus a absorção ótica. Nesta cauda, a absorção ótica cai a zero, em uma região normalmente transparente, em sólidos cristalinos [9]. Esta, então chamada região de Urbach, é atribuída à presença de estados eletrônicos deslocalizados próximos às bandas de valência ou de condução, em semicondutores amorfos [17]. Na região de alta energia da curva de absorção, a energia do "gap" ótico é relacionada à absorbância pela equação abaixo. Materiais com estas características podem apresentar propriedades como a fotoluminescência.

$$
\mathrm{h} v \alpha \propto\left(\mathrm{h} v-\mathrm{E}_{\mathrm{g}}^{\mathrm{opt}}\right)^{2}
$$

em que $\alpha$ é a absorbância, h a constante de Planck, $v$ a freqüência e $E_{\mathrm{g}}^{\text {opt }}$ o "gap" ótico da banda ("gap" de Tauc) [11].

A propriedade fotoluminescente observada para vários materiais (Fig. 5), como titanatos $\left(\mathrm{PbTiO}_{3}, \mathrm{BaTiO}_{3}\right.$ e $\left.\mathrm{SrTiO}_{3}\right)$, zirconatos $\left(\mathrm{PbZrO}_{3}\right.$ e $\left.\mathrm{SrZrO}_{3}\right)$, molibdato de estrôncio $\left(\mathrm{SrMoO}_{4}\right)$ e tungstato de estrôncio $\left(\mathrm{SrWO}_{4}\right)$, pode ser explicada, considerando-se a presença de cátions formadores e modificadores de rede no óxido metálico [12].

Óxidos formadores de rede apresentam basicamente ligações com natureza covalente e podem formar redes tridimensionais com o oxigênio. $\mathrm{O}$ fenômeno fotoluminescente pode ser previsto para estes formadores de rede, cuja escolha deve ser baseada em cátions metálicos, os quais formem, preferencialmente, complexos oxiânions pelo íon oxigênio em uma coordenação octaédrica ou tetraédica, com o átomo de oxigênio simétrico (Fig. 6). Oxiânions formados por Ti, Zr, Mo e W são bons candidatos [13].

Cátions metálicos que formem preferencialmente ligações iônicas com o oxigênio, tais como $\mathrm{Ba}, \mathrm{Sr}$ e $\mathrm{Pb}$, podem ser empregados como modificadores de rede. Modificadores de rede promovem a estabilização e a formação de "clusters" na rede tridimensional [10]. Baseado neste conceito, para a obtenção de materiais com propriedade fotoluminescente, é necessário que estes apresentem uma estrutura similar à mostrada na Fig. 7.

Em recente artigo [18] foi observado que, após a excitação

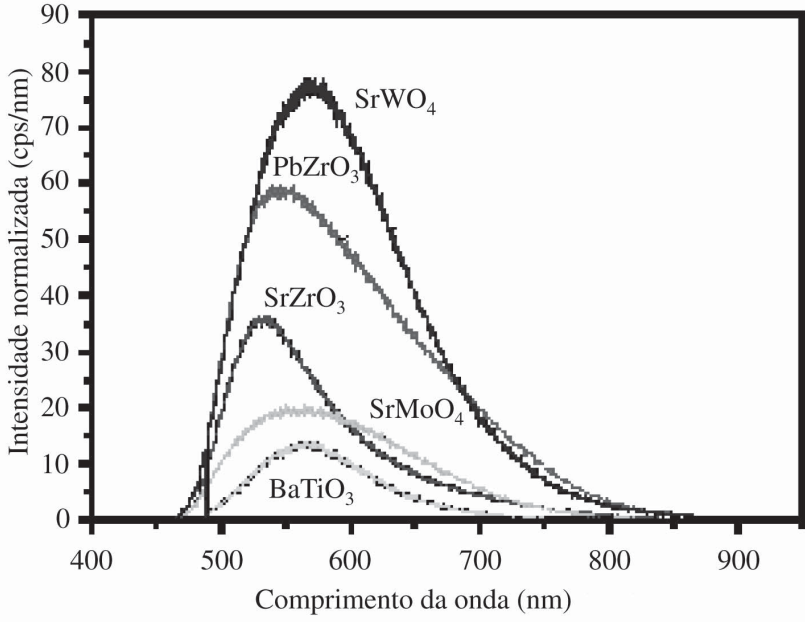

Figura 5: Espectros de fotoluminescência para diferentes cátions metálicos formadores de rede [10].

[Figure 5: Different photoluminescent spectra for different metallic net former cations [10].]

de um laser, titanatos amorfos obtidos pelo método Pechini emitiram bandas na faixa de freqüência do visível, à temperatura ambiente. Esta emissão pode estar associada à presença dos"“clusters" de $\mathrm{TiO}_{6}$ e $\mathrm{TiO}_{5}$ que suportam uma transferência de carga e aumentam os novos níveis nas bandas de valência (oxigênio) e de condução (metal) que são diretamente relacionados a fotoluminescência no visível.

O método Pechini tem se mostrado um forte aliado na pesquisa de fotoluminescência em sólidos amorfos [5-18,1519]. A síntese com homogeneidade em escala atômica, formação de partículas nanométricas, reprodutibilidade, entre

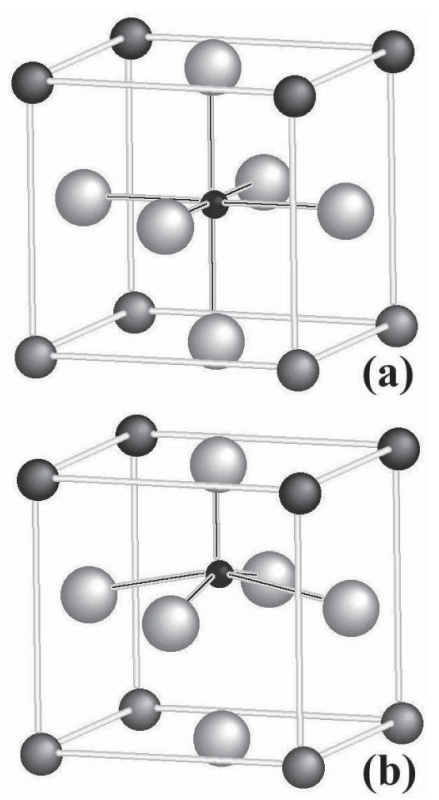

Figura 6: Poliedro de coordenação (a) octaédrica e (b) tetraédrica. [Figure 6: Polyhedrons of (a) octahedral and (b) tetrahedral coordination.] 


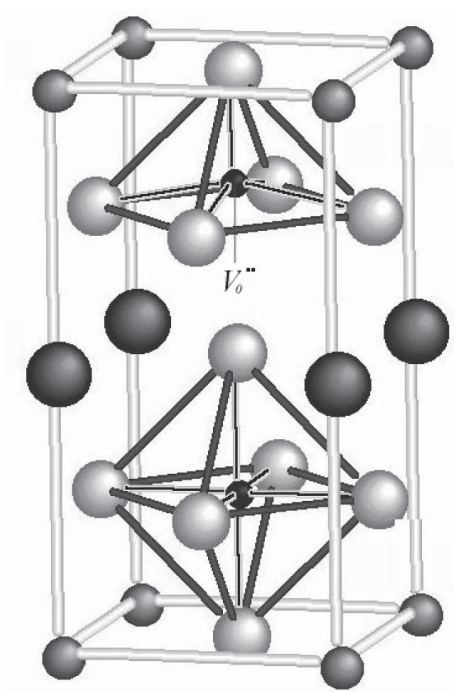

Figura 7: Representação 3D de um "cluster" de um material amorfo, $\mathrm{TiO}_{5}-\mathrm{TiO}_{6}[10]$

[Figure 7: $3 \mathrm{D}$ amorphous cluster material representation, $\mathrm{TiO}_{5}-$ $\left.\mathrm{TiO}_{6}[10].\right]$

outras vantagens deste método químico de síntese de óxidos cerâmicos multicomponentes, contribuem para este sucesso.

Em recente publicação [19], foi realizado um estudo teórico-experimental do papel dos modificadores de rede nas propriedades fotoluminescentes de titanatos amorfos e cristalinos, obtidos pelo método Pechini. Nesse trabalho, a propriedade fotoluminescente do $\mathrm{CaTiO}_{3}$ está relacionada ao estado amorfo deste óxido (Fig. 8).

Como esta propriedade está associado à desordem estrutural da fase inorgânica, o espectro fotoluminescente é mais intenso quando o material é submetido por mais tempo ao tratamento térmico à baixas temperaturas (o carbono é eliminado sem que haja cristalização). Isto é uma forte indicação que a fase desordenada (fase amorfa) é responsável

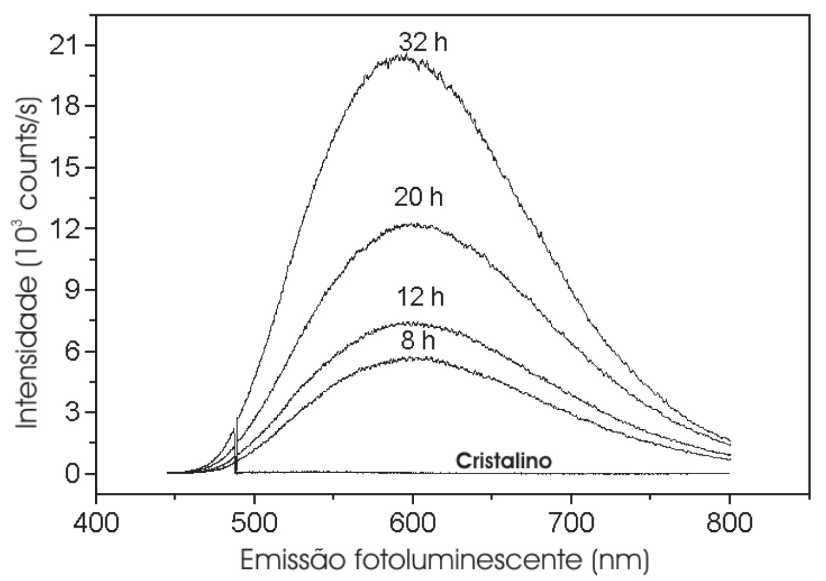

Figura 8: Espectros fotoluminescentes de $\mathrm{CaTiO}_{3}$ amorfo e cristalino obtidos pelo método Pechini, calcinados a 300 e $600^{\circ} \mathrm{C}$, respectivamente, por diferentes intervalos de tempo [19].

[Figure 8: Photoluminescent spectra of amorphous and crystalline $\mathrm{CaTiO}_{3}$ obtained by the Pechini method, calcinated at 300 and $600^{\circ} \mathrm{C}$, respectively, for different intervals of time [19].]

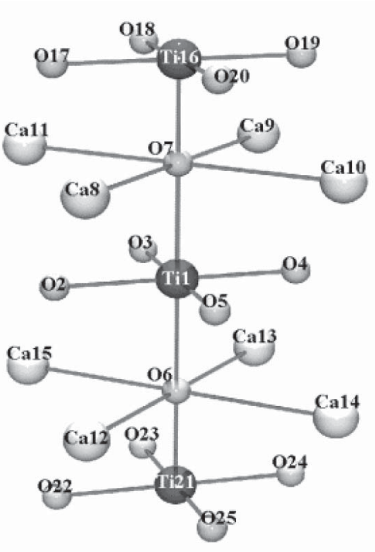

(a)

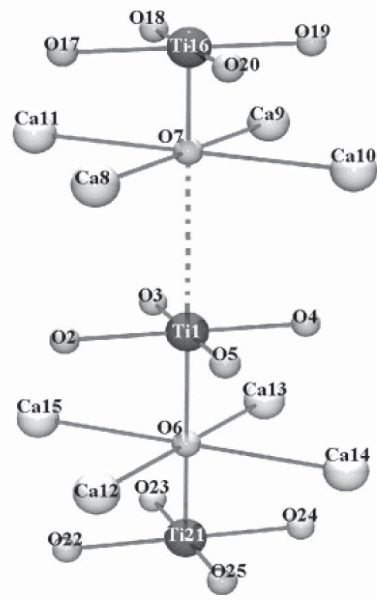

(b)
Figura 9: Representação do "cluster" $\mathrm{Ca}_{8} \mathrm{Ti}_{3} \mathrm{O}_{12}$ : (a) "cluster" cristalino e (b) "cluster" amorfo [19].

[Figure 9: Representation of $\mathrm{Ca}_{8} \mathrm{Ti}_{3} \mathrm{O}_{12}$ cluster: (a) crystalline cluster and (b) amorphous cluster [19].]

pela fotoluminescência [19]. Com o aumento da temperatura de calcinação, a ordem começa a aumentar a médio e longo alcance, logo as transições elétron-buraco sofrem uma diminuição, dessa forma diminuindo a intensidade do sinal fotoluminescente.

Várias discussões têm sido geradas na comunidade científica, na definição do estado amorfo. O estado amorfo possui uma desordem estrutural a longo alcance e uma ordem a curto alcance. Esta última pode estar associada às emissões fotoluminescentes de óxidos obtidos pelo método Pechini,

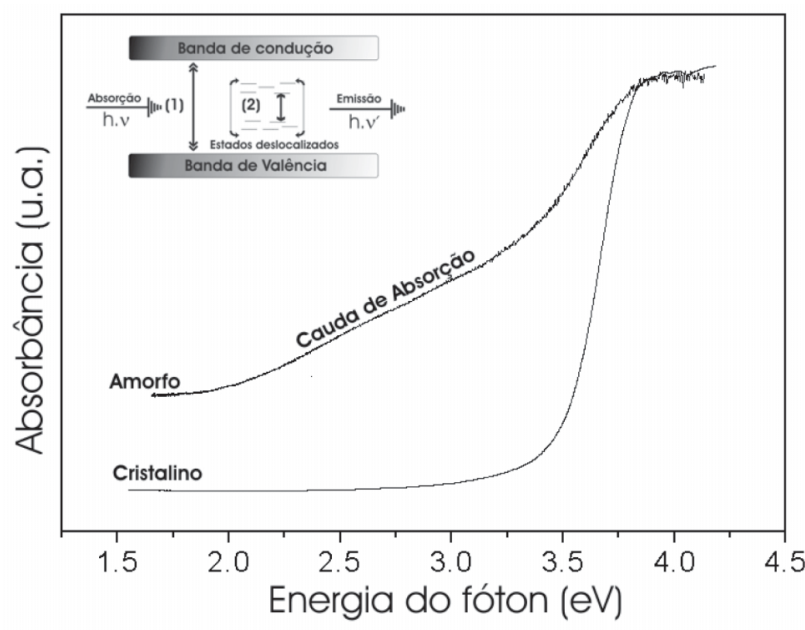

Figura 10: Espectro de absorbância de $\mathrm{CaTiO}_{3}$ amorfo e cristalino; (1) processo de recombinação direta da energia do "gap", o que é improvável para sólidos cristalinos; (2) provável energia de "gap" para o processo de recombinação [19].

[Figure 10: Absorbance spectrum of amorphous and crystalline $\mathrm{CaTiO}_{3}$; (1) process of direct recombination of the energy gap, that it is improbable for crystalline solids; (2) probable energy gap for the recombination process [19].] 


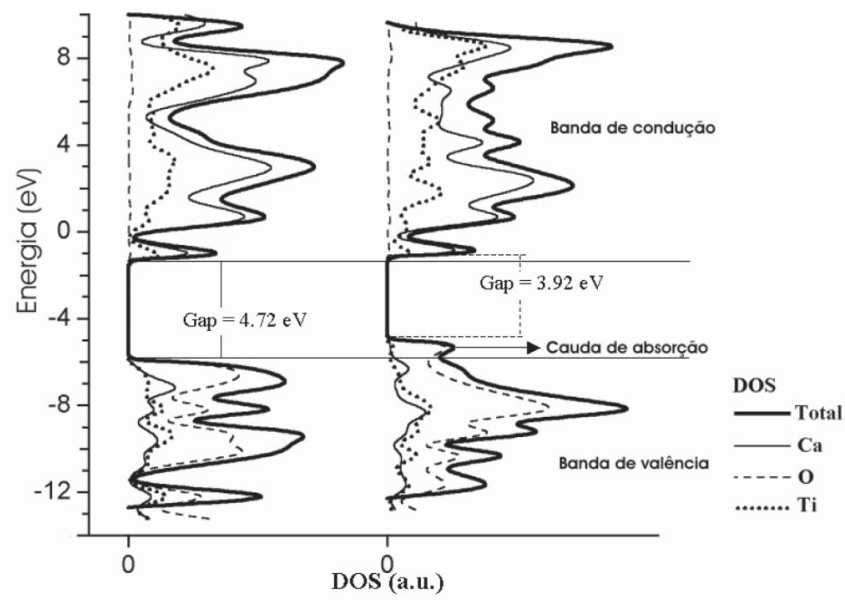

Figura 11: Densidade total dos estados para o $\mathrm{CaTiO}_{3}$ amorfo e cristalino [19]. [Figure 11: Total density of the states of the amorphous and crystalline $\mathrm{CaTiO}_{3}$ [19].]

que leva a um rígido controle estequiométrico e pode estar criando defeitos aleatórios e homogêneos, ou seja, uma ordem de defeitos a curto alcance.

A partir de cálculos teóricos, utilizando simulações do estado cristalino e amorfo do $\mathrm{CaTiO}_{3}$ (Fig. 9), foi observado que a formação de um "cluster" amorfo, pelo deslocamento de $\mathrm{O}(7)$, introduz níveis eletrônicos deslocalizados no "gap", o que também foi evidenciado experimentalmente pela formação de uma cauda de absorção (Fig. 10).

A partir da curva de densidade de estados (DOS), foi avaliada a contribuição de cada átomo desta configuração, na formação das bandas de valência e de condução [19] (Fig. 11).

A participação dos orbitais do oxigênio é bem mais efetiva na formação da banda de valência e a contribuição dos orbitais do cálcio e titânio é mais efetiva na banda de condução. A participação dos orbitais do cálcio - modificador de rede - na banda de condução é mais efetiva do que a do titânio - formador de rede [19]. O "cluster" cristalino apresenta um" "gap" maior do que o "cluster" amorfo, o que está em concordância com os dados experimentais de absorbância.

O diagrama dos níveis energéticos e de distribuição de cargas para o $\mathrm{CaTiO}_{3}$ revelam a energia dos orbitais de cada átomo e a distribuição de carga nos orbitais moleculares do Ti (Fig. 12).

A banda de condução do $\mathrm{CaTiO}_{3}$, tem a participação dos orbitais $d$ e $s$ do titânio e $s$ do cálcio e a banda de valência dos orbitais $2 p$ do oxigênio. Estes resultados teóricos indicam que existe uma transferência de carga entre os poliedros de coordenação $\mathrm{TiO}_{6}$ e $\mathrm{TiO}_{5}[19]$.

Foi sugerida que a fotoluminescência da estrutura desordenada (amorfa) do $\mathrm{CaTiO}_{3}$ está relacionada à recombinação radiativa entre elétrons e buracos, o que é refletido na cauda de absorção [19].

\section{CONCLUSÕES}

O estudo teórico-experimental, aliado ao rigor estequiométrico e a flexibilidade do método Pechini, estão

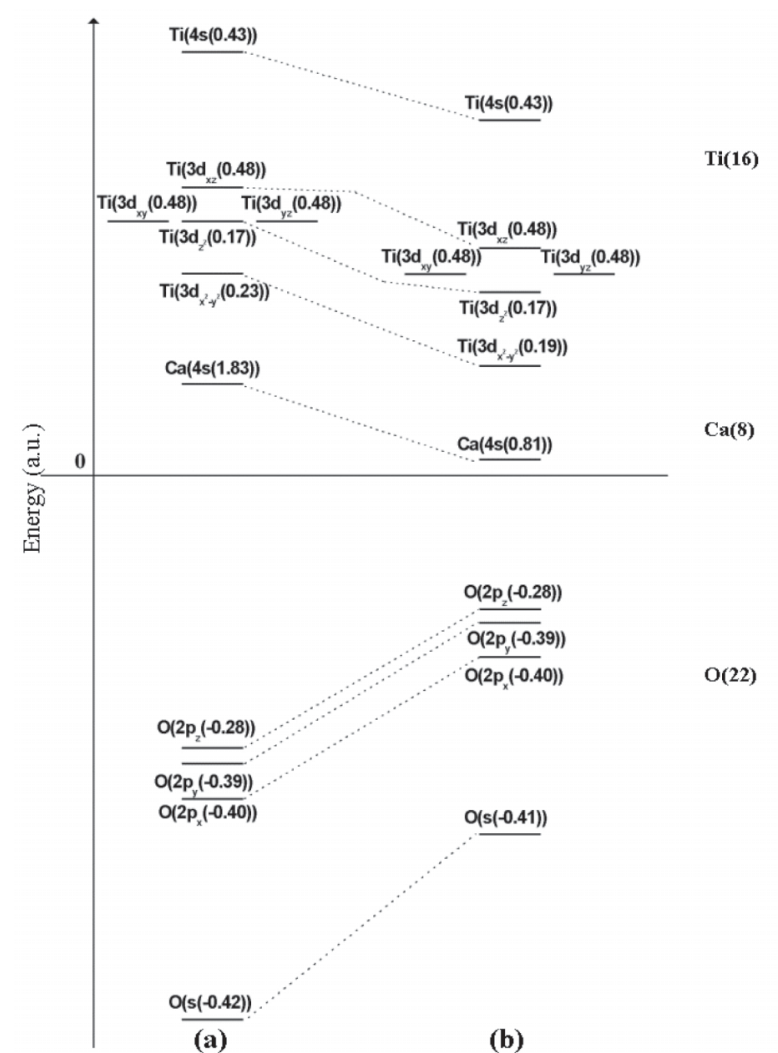

Figura 12: Diagrama dos níveis de energia e distribuição de cargas nos orbitais moleculares dos átomos do $\mathrm{CaTiO}_{3}$; a) cristalino e b) amorfo [19]. [Figure 12: Diagram of the energy levels and orbital charge distribution in atomic orbitals of the $\mathrm{CaTiO}_{3}$; a) crystalline and b) amorphous [19].]

contribuindo muito para o esclarecimento da origem da propriedade fotoluminescente em materiais com estrutura desordenada (amorfo). Deve-se ressaltar que existe uma transferência de carga entre os dois "clusters" $\mathrm{TiO}_{5}$ e $\mathrm{TiO}_{6}$, gerando novos níveis na banda de valência e de condução, diminuindo o "gap" na estrutura desordenada. Isto resulta na possibilidade de haver fotoluminescência à temperatura ambiente nos titanatos.

\section{AGRADECIMENTOS}

Os autores agradecem ao CNPq, à CAPES e à FAPESP/ CEPID, pelo financiamento deste trabalho.

\section{REFERÊNCIAS}

[1] A. R. Präss, Formas de radiação emissores de luz, $<$ http://www.fisicanet.terra.com.br/quantica $>$, acesso em $15 / 01 / 2003$.

[2] G. Blasse, J. Inorg. Nucl. Chem. 41 (1979) 639

[3] L. T. Canham, Appl. Phys. Lett. 57 (1990) 1046.

[4] D. Dimova-Malinvsca, Sol. Stat. Comm. 99 (1996) 641.

[5] E. R. Leite, N. L. V. Carreño, L. P. S. Santos, J. H. Rangel, L. E. B. Soledade, E. Longo, C. E. M. Campos, F. Lanciotti, P. S. Pizani, J. A. Varela, Appl. Phys. A 73 (2001) 1. [6] C. D. Pinheiro, F. M. Pontes, R. Magnani, P. S. Pizani, T. 
M. Boshi, J. A. Varela, E. R. Leite, E. Longo, Appl. Phys. A 75 (2002) 5.

[7] G. Blasse, B. C. Grabmaier, Luminescent Materials, Springer-Verlag, Berlin (1994).

[8] P. Goldberg, Luminescence of Inorganic Solids, Academic Press, New York (1966).

[9] D. L. Wood, J. Tauc, Phys. Rev. B 5 (1972) 3144.

[10] Fotoluminescência. <http//www.liec.ufscar.br $>$, acesso em 15/01/2003.

[11] Y. Tanabe, S. Sugano, J. Phys. Soc. Jap. 9 (1954) 753. [12] J. D. Lee, Química Inorgânica Não Tão Concisa, Edgard Blücher Ltda, $4^{\text {a }}$ Ed., S. Paulo (1997).

[13] P. T. Diallo, K. Jeanlouis, P. Boutinaud, R. Mahiou, J. C. Cousseins, J. Alloys \& Comp. 323-324 (2001) 218.
[14] B. Bouma, G. Blasse, J. Phys. Chem. Sol. 56 (1995) 261. [15] F. M. Pontes, E. R. Leite, E. Longo, J. A. Varela, P. S. Pizani, C. E. M. Campos, F. Lanciotti, Adv. Mater. Opt. Electr. 102 (2000) 81.

[16] P. S. Pizani, E. R. Leite, F. M. Pontes, E. C. Paris, J. H. Rangel, E. Lee, E. Longo, P. Delega, J. A. Varela, Appl. Phys. Lett. 77 (2001) 824.

[17] M. Capizzi, A. Frova, Phys. Rev. Lett. 25 (1970) 1298. [18] E. R. Leite, F. M. Pontes, E. C. Paris, C. A. Paskocimas, E. Lee, E. Longo, P. S. Pizani, J. A. Varela, V. Mastelaro, Adv. Mater. Opt. Electr. 106 (2000) 235.

[19] F. M. Pontes, C. D. Pinheiro, E. Longo, E. R. Leite, S. R. Lazaro, J. A. Varela, P. S. Pizani, T. M. Boschi, F. Lanciotti, Mater. Chem. Phys. 78 (2002) 227.

(Rec. 28/08/03, Ac. 31/10/03) 